\title{
Analysis of Cargo Sway Characteristics of Tower Cranes
}

\author{
Mingxiao Dong ${ }^{\mathrm{a}}$, Bo Pang ${ }^{\mathrm{b}}$ and Jiyong Wang ${ }^{\mathrm{c}}$ \\ Mechatronic Engineering School, Shandong Jianzhu University, Jinan, 250101, China \\ amxdong@sdjzu.edu.cn, bpl-plcn@sdjzu.edu.cn, cwjyhjn@sdjzu.edu.cn
}

Keywords: Tower crane; cargo; sway.

Abstract. To study the cargo motion and sway characteristics of tower cranes to find effective methods to suppress the cargo sway and to position the cargo, we modeled the cargo sway, and then analyzed the equilibrium states of the cargo sway. The research results show that the center line of the cargo pendulum is declining, just as the cargo oscillates in the inclined gravity field because of there are inertia forces and the centrifugal forces.

\section{Introduction}

Tower cranes have 3 kinds of basic working condition that are the transition, the rotation and the hoisting movement. Rotary motion causes centrifugal force and inertia force in the varying direction, so that the analysis of the dynamic characteristics is more complex. In this paper, the dynamic characteristics of the tower cranes at the working condition of the transition, the rotation, and the hoisting movement at the same time. The nonlinear models of the cargo sway were set in the non-inertia reference system and the equilibrium states of the cargo are calculated. The models are linear near the equilibrium states and the cargo sway characteristics are simulated ${ }^{[1-3]}$.

\section{Non-Linear Models of Cargo Sway}

Tower cranes transport cargoes by rotation of crane jib and trolley motion along the jib. According to the motion characteristics, we set up a polar coordinate system $\left\{\mathrm{e}_{\rho}, \mathrm{e}_{\psi}\right\}$ whose coordinate origin is located at the intersection of the rotary center line of the tower body and the rotary surface of the crane jib. The inertia force, centrifugal force, and external disturbance causes the cargoes to oscillate in space pendulum and hoisting motion changes the length of the pendulum. Based on this, we set up spherical coordinate system $\left\{\mathrm{e}_{l}, \mathrm{e}_{\theta}, \mathrm{e}_{\phi}\right\}$ whose coordinate origin located at the suspension point of the hoisting cable and the suspension point moves with the trolley and rotates synchronously with the $\mathrm{jib}^{[4,5]}$. The coordinate system is shown in Fig. 1.

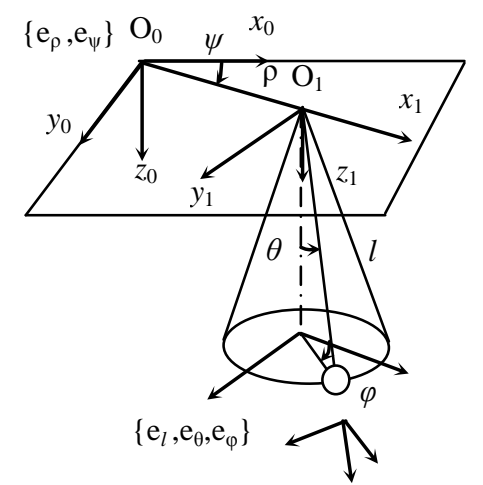

Fig.1 Tower crane coordinate system

The payload suspension point is located at $(\rho, \psi)$ in the polar coordinates, where $\rho$ and $\psi$ respectively denote the displacement of the trolley and the rotation angle of the jib. The position of the payload in the non-inertia spherical coordinate system is described by three generalized coordinates $(l, \theta, \varphi)$. 
Analysis of the External Forces Acting on the Cargo. The external forces acting on the cargo include the gravity $G$ and hoisting cable tension force $T$, where the gravity $G$ is decomposed as $F_{g \theta}$ and $F_{g \phi}$ along $\mathrm{e}_{\theta}$ direction and $\mathrm{e}_{\phi}$ direction and tension force $T$ along the direction of hoisting cable not affects the movement of the cargo in $\mathrm{e}_{\theta}$ direction and $\mathrm{e}_{\varphi}$ direction. $F_{g \theta}$ and $F_{g \phi}$ are following.

$$
\begin{gathered}
F_{g \theta}=-m g \sin \theta . \\
F_{g \phi}=0 .
\end{gathered}
$$

Analysis of the Inertia Forces. When the trolley moves along the jib, the consultant force of the inertial force and the air resistance (the air resistant coefficient is $f$ ) is $F_{\rho}$, which is respectively decomposed as $F_{\theta \rho}$ and $F_{\phi \rho}$ along $\mathrm{e}_{\theta}$ direction and $\mathrm{e}_{\varphi}$ direction.

$$
\begin{aligned}
& F_{\theta \rho}=-(m \ddot{\rho}+f \dot{\rho}) \cos \theta \cos \phi . \\
& F_{\phi \rho}=(m \ddot{\rho}+f \dot{\rho}) \sin \phi .
\end{aligned}
$$

The convected inertial force, centrifugal force, and the air resistance caused by jib rotaion can be decomposed as $F_{\theta \psi}$ and $F_{\phi \psi}$ respectively along the $\mathrm{e}_{\theta}$ and $\mathrm{e}_{\varphi}$ direction. Due to the amplitude of cargo sway with respect to the gyration radius of the cargo suspension point is much smaller during calculating the inertia force, centrifugal force and air resistance caused by the rotary motion, the gyration radius in $\mathrm{e}_{\theta}$ direction can be neglected.

$$
\begin{gathered}
F_{\theta \psi}=-(m \rho \ddot{\psi}+f \rho \dot{\psi}) \cos \theta \sin \phi+m \rho \dot{\psi}^{2} \cos \theta \cos \phi \\
F_{\phi \psi}=-(m \rho \ddot{\psi}+f \rho \dot{\psi}) \cos \phi-m \rho \dot{\psi}^{2} \sin \phi \quad . .
\end{gathered}
$$

Analysis of Coriolis Forces. Transition and rotary motion produce Coriolis force that is decomposed as $F_{\theta \rho \mu}$ and $F_{\phi \rho \psi}$ respectively along $\mathrm{e}_{\theta}$ direction and $\mathrm{e}_{\varphi}$ direction. They are

$$
\begin{gathered}
F_{\theta \rho \psi}=2 m \dot{\rho} \dot{\psi} \cos \theta \sin \phi . \\
F_{\phi \rho \psi}=2 m \dot{\rho} \dot{\psi} \cos \phi .
\end{gathered}
$$

Coriolis force $F_{l \theta}=2 m i \dot{\theta}$, along $\mathrm{e}_{\theta}$ direction, results from the hoisting motion and the sway of the cargo in $\mathrm{e}_{\theta}$ direction. The Coriolis force $F_{l \phi}$ induced from the hoisting movement and the oscillation in $\mathrm{e}_{\varphi}$ direction is $F_{l \phi}=2 m i \dot{\phi} \sin \theta$. The oscillations in $\mathrm{e}_{\theta}$ direction and $\mathrm{e}_{\varphi}$ direction produce Coriolis force $F_{\theta \phi}$ in $\mathrm{e}_{\varphi}$ direction, $F_{\theta \phi}=2 m l \dot{\theta} \dot{\phi} \cos \theta$.

Effect of Wind Loads. Given the wind speed in the ox direction and oy direction are respectively $u_{x}$ and $u_{y}$, the wind resistance coefficient is $f$, the wind loads are $f_{u x}$ and $f_{u y}$. The two loads are decomposed as $F_{\theta u}$ and $F_{\phi u}$ along the $\mathrm{e}_{\theta}$ direction and $\mathrm{e}_{\varphi}$ direction.

$$
\begin{gathered}
F_{\theta u}=\left[f u_{x} \cos (\phi+\psi)+f u_{y} \sin (\phi+\psi)\right] \cos \theta . \\
F_{\phi u}=-f u_{x} \sin (\phi+\psi)+f u_{y} \cos (\phi+\psi) .
\end{gathered}
$$

The circular motion of the cargo in $\mathrm{e}_{\varphi}$ direction produces centrifugal force $F_{\phi}$ whose component force is $F_{\phi}=m l \dot{\phi}^{2} \sin \theta \cos \theta$ along the $\mathrm{e}_{\theta}$ direction. The sway of Cargo in $\mathrm{e}_{\theta}$ direction causes the centrifugal force along the direction of hoisting cable.

The air resistance of the cargo movement in $\mathrm{e}_{\theta}$ direction, $F_{f \theta}=-f l \dot{\theta}$, the air resistance of the cargo movement in $\mathrm{e}_{\varphi}$ direction $F_{f \phi}=-f l \sin \theta \dot{\phi}$. The inertial force from hoisting motion along the hoisting cable direction does not have an impact on the movements in $\mathrm{e}_{\theta}$ direction and $\mathrm{e}_{\varphi}$ direction. According to the relationship of the oscillating torque balance in $\mathrm{e}_{\theta}$ direction and in $\mathrm{e}_{\varphi}$ direction in the non-inertia reference system, the dynamic equations of cargo sway are modeled as following.

$$
\begin{aligned}
& m l^{2} \ddot{\theta}=\sum M=-m g l \sin \theta+m l^{2} \dot{\phi}^{2} \sin \theta \cos \theta-(m l \ddot{\rho}+f l \dot{\rho}) \cos \theta \cos \phi \\
& -(m \rho l \ddot{\psi}+f \rho l \dot{\psi}) \cos \theta \sin \phi+m \rho l \dot{\psi}^{2} \cos \theta \cos \phi-2 m l i \dot{\theta}-2 m l \dot{\rho} \dot{\psi} \cos \theta \sin \phi . \\
& -f l^{2} \dot{\theta}+\left[f u_{x} \cos (\phi+\psi)+f u_{y} \sin (\phi+\psi)\right] l \cos \theta
\end{aligned}
$$




$$
\begin{aligned}
& m l^{2} \sin ^{2} \theta \ddot{\phi}=\sum M=-2 m l^{2} \dot{\theta} \dot{\phi} \sin \theta \cos \theta+(m l \ddot{\rho}+f l \dot{\rho}) \sin \theta \sin \phi \\
& -(m \rho l \ddot{\psi}+f \rho l \dot{\psi}) \sin \theta \cos \phi-m \rho l \dot{\psi}^{2} \sin \theta \sin \phi-2 m l i \dot{\phi} \sin ^{2} \theta-2 m l \dot{\rho} \dot{\psi} \sin \theta \cos \phi . \\
& -f l^{2} \dot{\phi} \sin ^{2} \theta+\left[-f u_{x} \sin (\phi+\psi)+f u_{y} \cos (\phi+\psi)\right] l \sin \theta
\end{aligned}
$$

The Eq. (1) and Eq. (2) are the models of cargo sway under the condition of the tower crane carrying out the transition, rotation, and hoisting motion at the same time.

\section{Linear Models of Cargo Sway}

The equilibrium states of the tower crane are achieved by ordering $\dot{\theta}(t)=0, \ddot{\theta}(t)=0, \dot{\phi}(t)=0, \ddot{\phi}(t)=0$. The linear models are obtained by inputting a small disturb in the vicinity of the system equilibrium states, ignoring the high order terms, and keeping the first order term.

$$
\begin{gathered}
\phi_{e}=\tan ^{-1}\left(-\frac{\rho \ddot{\psi}+\dot{\rho} \dot{\psi}}{\rho \dot{\psi}^{2}-\ddot{\rho}}\right) . \\
\theta_{e}=\tan ^{-1} \frac{a}{g} .
\end{gathered}
$$

Where, $\sin \phi_{e}=-\frac{\rho \ddot{\psi}+\dot{\rho} \dot{\psi}}{a}, \cos \phi_{e}=\frac{\rho \dot{\psi}^{2}-\ddot{\rho}}{a}, a=\sqrt{(\rho \ddot{\psi}+\dot{\rho} \dot{\psi})^{2}+\left(\rho \dot{\psi}^{2}-\ddot{\rho}\right)^{2}}$.

The equilibrium state $\theta_{\mathrm{e}}$ expresses the angle between the hoisting cable and the plumb line through the hanging point of the cargo and $\varphi_{\mathrm{e}}$ expresses the angle between the hoisting cable and the plane where the plumb line exists. $\theta_{\mathrm{e}}$ is as a result of the interaction of inertia force, centrifugal force and Coriolis force. The magnitude of $\varphi_{\mathrm{e}}$ is the ratio of the consultant force of rotary inertia force and Coriolis force over the consultant force of rotating centrifugal force and inertial force. Due to the joint action of the inertia force, the centrifugal force and the Coriolis force, so that oscillating center line of the cargo deflects, just as the cargo does space pendulum in inclined gravity field as shown as in Fig. 2.

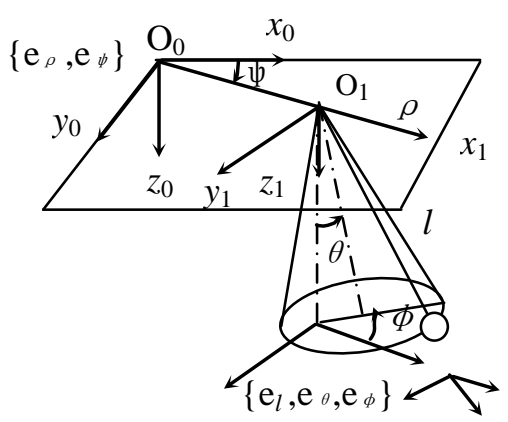

Fig. 2 Cargo sway when oscillation center line inclines

Linear models in the vicinity of the system equilibrium states are following.

$$
\begin{aligned}
& l \delta \ddot{\theta}+2 i \delta \dot{\theta}+\sqrt{g^{2}+a^{2}} \delta \theta=-\cos \theta_{e}\left(\cos \phi_{e} \delta \ddot{\rho}+\sin \phi_{e} \delta \ddot{\psi}\right) . \\
& l \delta \ddot{\phi}+2 i \delta \dot{\phi}+\sqrt{g^{2}+a^{2}} \delta \phi=\left(\sin \phi_{e} \delta \ddot{\rho}-\rho \cos \phi_{e} \delta \ddot{\psi}\right) / \sin \theta_{e} .
\end{aligned}
$$

Eq. (15) and Eq. (16) are second-order differential equation about the pendulum angle $\theta$ and $\varphi$. The frequency of the sway is decided by the ratio of the synthetic acceleration over the hoisting cable length. And the sway amplitude is relative with transition inertial force and rotary inertia force. So oscillating frequency and amplitude can be controlled by the motion acceleration of the mechanisms.

\section{System Simulation}

The cargo sway characteristics are carried out by the simulation test for the QTZ135tower crane controlled by frequency converter. Given the following conditions, the cargo mass $m=2000 \mathrm{~kg}$, the rated motion speed of the trolley $v=1 \mathrm{~m} / \mathrm{s}$, the rated rotary speed of the rotary mechanism $\omega=0.0268 \mathrm{rad} / \mathrm{s}$, the rated hoisting speed of hoisting mechanism is $1 \mathrm{~m} / \mathrm{s}$. Speed up for $4 \mathrm{~s}$ based on the trapezoidal speed curve before reaching the rated speed, run for $92 \mathrm{~s}$ at a constant speed, decelerate 
for $4 \mathrm{~s}$ before stopping. The system initial states are 0. Fig. 3 shows the cargo sway angular displacement and velocity response curve when the crane transports and rotates at the same time and its oscillation amplitude and period is constant. Along with the transportation carrying on, the centrifugal force increases gradually, $\theta(t)$ deviates from the equilibrium position, the oscillating center line inclines and is no longer along the vertical direction.
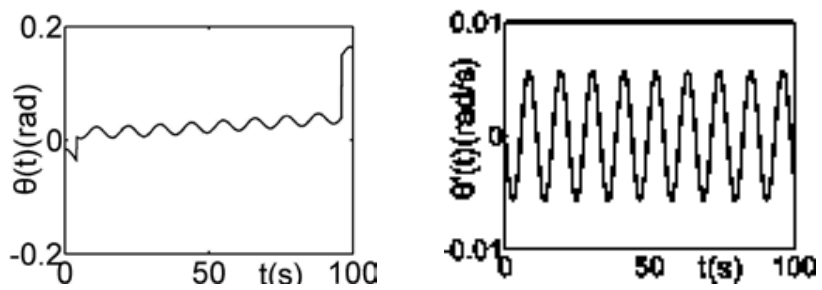

(a) Cargo sway angle $\theta(t)$

(b) Cargo sway angular velocity $\dot{\theta}(t)$
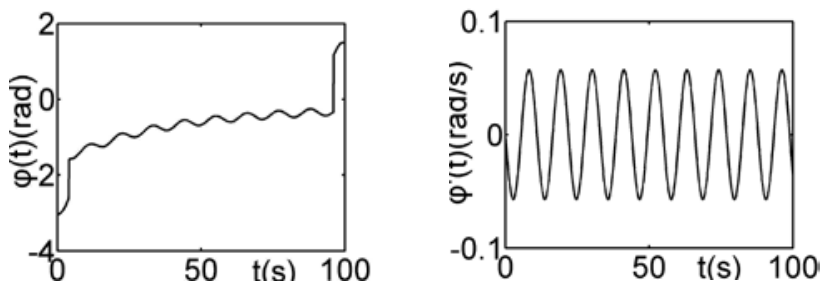

(c) Cargo sway angle $\phi(t)$

(d) Cargo sway angular velocity $\dot{\phi}(t)$

Fig. 3 The cargo sway angle and angular velocity

\section{Conclusion}

In this paper, the nonlinear models of cargo sway are set up when the tower crane translates, rotates, and hoists and the models are linear near the equilibrium states to reveal that the characteristics of the cargo sway. Due to the inertia force or the centrifugal force, the center line of the cargo sway is tilted, just as the cargo does a space pendulum motion in the tilted gravity field whose sway model is the linear model and the deflection of the sway center line is the balance state values.

\section{Acknowledgements}

This work was financially supported by the National Natural Science Foundation of China (Grant No. 51475277).

\section{References}

[1] Andreas Rauh, Luise Senkel, Jovanka Gebhardt, Harald Aschemann. Stochastic Methods for the Control of Crane Systems in Marine Applications. 2014 European Control Conference (ECC), June 24-27, 2014, Strasbourg, France (2014), p2998-3003.

[2] Jisup Yoon, Shelley Nation, William Singhose, and Joshua Eric Vaughan. Control of Crane Payloads That Bounce During Hoisting. IEEE Transactions on Control Systems Technology, Vol. 22, No. 3, May (2014), p1233-1238.

[3] Willian Singhose, lisa Porter, Michael Kenison et al. Effects of hoisting on the input shaping control of gantry cranes. Control Engineering Practice, Vol. 8, No 10 (2000), p1159-1165.

[4] Mingxiao Dong. Research on Time-delayed Control Theory and Its Application to Realization of Crane Automation. Xi'an, Xi’an Jiaotong University. 2005,12.

[5] Mingxiao Dong. Time-delayed filter theory and engineering application. Beijing, Science Press. 2008,3. 